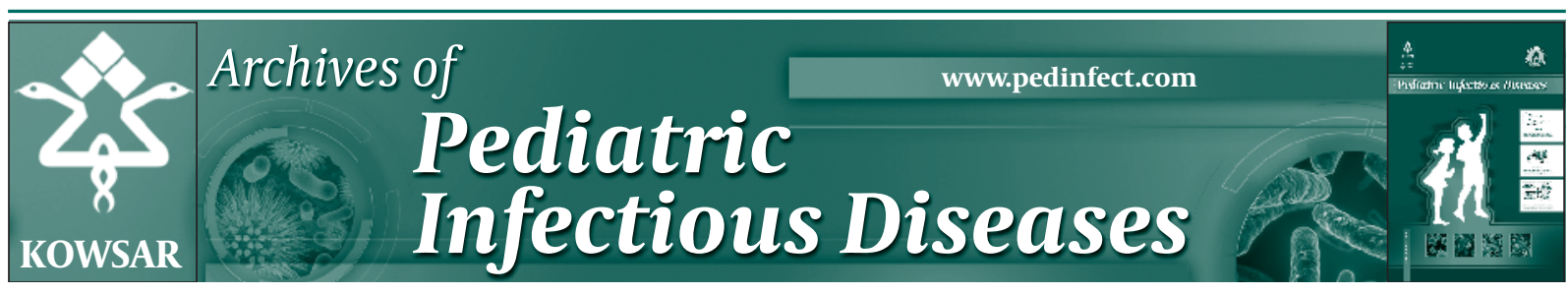

\title{
A Case Report; Purulent Meningitis Due to Serotype 2 of Streptococcus pneu- moniae
}

\author{
Selda Hancerli Torun ${ }^{1,}$, Bahar Calıskan ${ }^{1}$, Ayper Somer ${ }^{1}$, Nuran Salman ${ }^{1}$, Nezahat Guler ${ }^{2}$ \\ ${ }^{1}$ Istanbul Medical Faculty Department of Pediatric Infection Disease, Istanbul, Turky \\ ${ }^{2}$ Istanbul Medical Faculty Department of Microbiology, Istanbul, Turky \\ *Corresponding author: Selda Hancerli Torun, Istanbul Medical Faculty, Department of Pediatric Infection Disease, Istanbul, Turky, Tel.: +90-2126333540, \\ Fax:+90-2122630417, E-mail: seldahancerli@hotmail.com.
}

\begin{abstract}
A B S T R A C T
Children under 2 years of age suffer from some of the highest rates of invasive pneumococcal disease (IPD) in the world. (Turkey) Rates of IPD had a decline after statewide introduction of the 7-valent pneumococcal conjugate vaccine (PCV7) in 2008. Introduction of PCV7, PCV10 and PCV13 vaccines resulted in elimination of IPD caused by vaccine serotypes, but was followed by increasing rates of IPD caused by nonvaccine serotypes. Although the heptavalent vaccine has demonstrated its ability to reduce the incidence of pneumococcal disease, its efficacy is limited due to the restricted number of serotypes included. We report a case involving a 3-monthold girl who developed meningitis with Streptococcus pneumoniae serotype 2 despite the use of heptavalent conjugate vaccine.
\end{abstract}

Keywords: Meningitis, Pneumococcal; Streptococcus pneumoniae

Copyright @ (2013, Kowsar Corp.; Published by Kowsar Corp.

\section{Introduction}

Invasive pneumococcal disease (IPD) in children persists as a major cause of morbidity and mortality throughout the world. Rates of IPD had a decline after statewide introduction of the 7-valent pneumococcal conjugate vaccine (PCV7) in 2008. Introduction of PCV7, PCV10 and PCV13 vaccines resulted in elimination of IPD caused by vaccine serotypes, but was followed by increasing rates of
IPD caused by nonvaccine serotypes. Further surveillance on Streptococcus pneumoniae is important to understand the consequences of emerging nonvaccine serotypes and the additional serotypes that should be incorporated into the second-generation vaccines. We report a case of a 3-month-old girl with meningitis caused by serotype 2 of S. pneumoniae despite the use of heptavalent conjugate vaccine.

\footnotetext{
Article type: Case Report; Received: 25 Apr 2012, Revised: 11 Jul 2012, Accepted: 11 Mar 2013; DOI: 10.5812/pedinfect.5314

Implication for health policy/practice/research/medical education:

Children under 2 years of age suffer from some of the highest rates of invasive pneumococcal disease (IPD) in the world. (Turkey) Rates of IPD had a decline after state-wide introduction of the 7-valent pneumococcal conjugate vaccine (PCV7) in 2008. Introduction of PCV7, PCV10 and PCV13 vaccines resulted in elimination of IPD caused by vaccine serotypes, but was followed by increasing rates of IPD caused by nonvaccine serotypes. It is possible that serotype 2 may be an invasive strain with low morbidity if the proper treatment is applied. More case reports are needed to confirm this comment.
}

Please cite this paper as:

Torun SH, Calıskan B, Somer A, Salman N, Guler N. A Case Report; Purulent Meningitis Due to Serotype 2 of Streptococcus pneumoniae. Arch Pediatr Infect Dis. 2013;1(3): 144-6. DOI: 10.5812/pedinfect.5314 


\section{Case Report}

A previously healthy 3-months old girl was hospitalized with fever (up to 39,5oC), fatigue and lack of appetite during the last 2 days. On physical examination, she had pulsatile fontanelle with reduced interest around. The rest of the physical examination was unremarkable. The patient had received a PCV7 a month prior to her recent hospitalization. Routine laboratory tests revealed leukocytosis (13500/ $\mathrm{mm}^{3}$ ) and elevated C-reactive protein $(32 \mathrm{mg} / \mathrm{L})$. Cerebrospinal fluid analysis showed a low glucose level $(6 \mathrm{mg} / \mathrm{dL})$, elevated protein level (213 mg/dL), abundant leukocytes and gram-positive diplococcus in gram staining. The CSF culture specimen was positive for S.pneumoniae. Isolate was found to be susceptible to Penicillin, Erythromycin, Chlarithromycin, Cefotaxime and resistance to Cotrimoxazole according to clinical and laboratory standarts and institute (CLSI)'s guidelines. Serotype 2 was determined by the capsular swelling reaction (quelling) and latex agglutination.

The patient was put on Ceftriaxone and Vancomycin therapy based on the diagnosis of pneumococcal meningitis. Soon after her hospitalization she began to experience generalized tonic-clonic seizure and her fever was still not under control. Subdural collection and pathologic contrast enhancement were observed over the left frontoparietal region on cranial computed tomography scan (Figure 1). Subdural puncture was performed and 30 $\mathrm{mL}$ of purulent fluid was drained. She was followed on external drainage for about a month and during that time period antibiotherapy was continued. On the third day of external drainage of subdural space her body temperature was back to normal degrees and her convulsions disappeared. The patient underwent subduroperitoneal shunting on the 31th day of treatment. 10 days later she was discharged from hospital. To date, over the course of a follow-up period of more than 11 months, the patient has shown no neurological signs of relapse; normal hearing and neuromotor development have been observed.

\section{Discussion}

Here we describe a 3-month-old girl with meningitis due to S. pneumoniae serotype 2. Understanding the epidemiology and serotype distribution of pneumococcal disease is vital for reducing the burden of disease and its associated morbidity and mortality.

The fundamental empirical differentiation of pneumococcal isolates has been the unique serological identities of their capsular polysaccharides (1). At present, a total of 91 serologically distinct capsular polysaccharides have been described $(2,3)$. A subsequent meta-analysis incor porated seven diverse, globally distributed data sets from similarly matched pediatric IPD and carriagestrains to calculate serotype- and serogroup-specific invasiveness by using an odds ratio. These data supported the conclusion that pneumococcal serotypes differ in invasiveness and that serotypes and serogroups 1, 4, 5, 7, 14 and $18 \mathrm{C}$ are associated with invasive disease, whereas serotypes and serogroups 3, 6A, 6B, 15, 19 and 23 are associated with carriage (4). To the best of our knowledge invasive pneumococcal disease such as meningitis with serotype 2 has not been described in recent years.

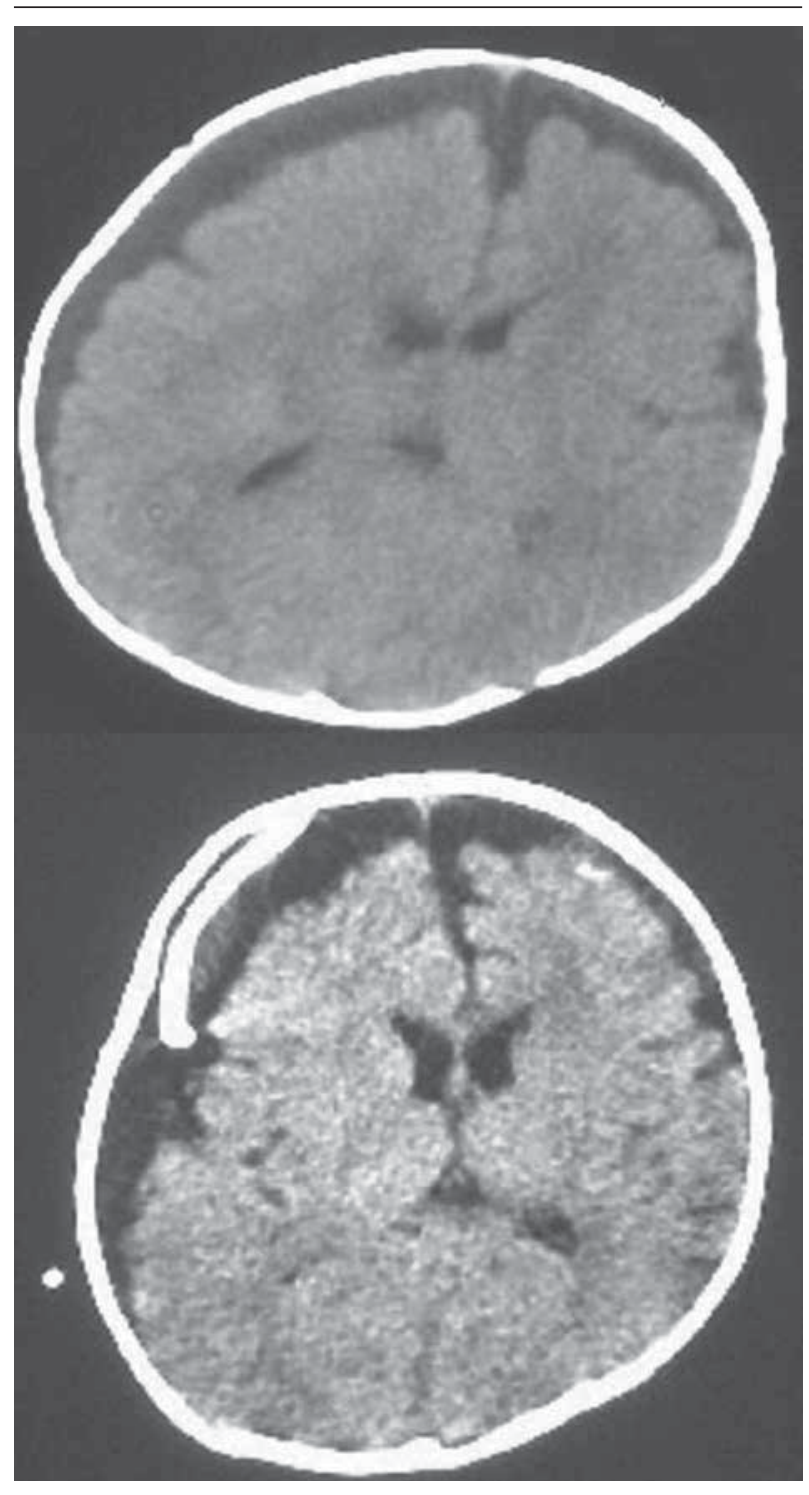

Figure 1. CT of Cranium

In Harboe et al's retrospective cohort study based on nationwide laboratory surveillance data on IPD collected uninterruptedly in Denmark during 1938-2007, changes in the reported incidence and trends of pneumococcal serotypes were explored using nonlinear regression analysis. The proportions of serotypes/groups 4 and 9 increased; the proportion of serotype $18 \mathrm{C}$ decreased; the proportions of serotypes $6,7 \mathrm{~F}, 14$, and $23 \mathrm{~F}$ remained stable; and serotype 2 nearly disappeared. Before the 1960s, serotypes 1, 2,3, and 5 presented peaks every 
2-3 years, becoming less frequent during the 1970s with peaks every 7-10 years. The epidemiology of IPD and single serotypes has constantly changed over the past 7 decades. PCV serotypes appeared to dominate the pneumococcal population(5).

Pneumococcal meningitis, although the least common of the syndromes, occurs primarily in young infants (6). The incidence of pneumococcal meningitis reported in Western Europe and the USA (8-9 per 100,000 children each year) was similar prior to the introduction of PCV7. In addition to the higher incidence rates of pneumococcal meningitis in industrializing countries, greater case fatality rates and neurological sequelae have been observed in these children compared to those of industrialized countries (7). Case-fatality rates excess of $50 \%$ have been reported from some African and Asian countries. $S$. pneumoniae is believed to contribute significantly to the 100,000-500,000 annual deaths from meningitis among children in industrializing countries. Our patient had meningitis with serotype 2 of Streptococcus pneumonia. Since full recovery has been observed in our case, it's possible that serotype 2 may be an invasive strain with low morbidity if the proper treatment is applied. More case reports are needed to confirm this comment.

\section{Acknowledgements}

There is no aknowledgments.

\section{Authors' Contributions}

None declared.

\section{Financial Disclosure}

We have no financial interests related to the material in the manuscript.

\section{Funding/Support}

There is no funding.

\section{References}

1. van Dam JE, Fleer A, Snippe H. Immunogenicity and immunochemistry of Streptococcus pneumoniae capsular polysaccharides. Antonie Van Leeuwenhoek. 1990;58(1):1-47.

2. Park IH, Pritchard DG, Cartee R, Brandao A, Brandileone MC, Nahm MH. Discovery of a new capsular serotype (6C) within serogroup 6 of Streptococcus pneumoniae. J Clin Microbiol. 2007;45(4):1225-33.

3. Kamerling JOHANNIS P, Thomasz A. Pneumococcal polysaccharides: a chemical view. In: Kamerling JOHANNIS P, Thomasz A, editors.Streptococcus pneumoniae, Molecular biology and mechanisms of disease.NY: M.Aliebert,Inc.,Larchmont; 2000. p. 81-114.

4. Brueggemann AB, Peto TE, Crook DW, Butler JC, Kristinsson KG Spratt BG. Temporal and geographic stability of the serogroupspecific invasive disease potential of Streptococcus pneumoniae in children. J Infect Dis. 2004;190(7):1203-11.

5. Harboe ZB, Benfield TL, Valentiner-Branth P, Hjuler T, Lambertsen $\mathrm{L}$, Kaltoft M, et al. Temporal trends in invasive pneumococcal disease and pneumococcal serotypes over 7 decades. Clin Infect Dis. 2010;50(3):329-37.

6. Kellner JD, Vanderkooi OG, MacDonald J, Church DL, Tyrrell GJ, Scheifele DW. Changing epidemiology of invasive pneumococcal disease in Canada, 1998-2007: update from the Calgary-area Streptococcus pneumoniae research (CASPER) study. Clin Infect Dis. 2009;49(2):205-12.

7. Greenwood B. The epidemiology of pneumococcal infection in children in the developing world. Philos Trans R Soc Lond B Biol Sci. 1999;354(1384):777-85. 